\title{
Propagation Characteristics of Oblique Incident Terahertz Wave in Nonuniform Dusty Plasma
}

\author{
Yunhua Cao, ${ }^{1}$ Haiying Li, ${ }^{1,2}$ Zhe Wang, ${ }^{1}$ and Zhensen $\mathrm{Wu}^{1,2}$ \\ ${ }^{1}$ School of Physics and Optoelectronic Engineering, Xidian University, Xian, Shaanxi 710071, China \\ ${ }^{2}$ Collaborative Innovation Center of Information Sensing and Understanding, Xidian University, Xi'an, Shaanxi 710071, China \\ Correspondence should be addressed to Haiying Li; lihy@xidian.edu.cn
}

Received 29 August 2016; Accepted 23 October 2016

Academic Editor: Atsushi Mase

Copyright (C) 2016 Yunhua Cao et al. This is an open access article distributed under the Creative Commons Attribution License, which permits unrestricted use, distribution, and reproduction in any medium, provided the original work is properly cited.

\begin{abstract}
Propagation characteristics of oblique incident terahertz wave from the nonuniform dusty plasma are studied using the propagation matrix method. Assuming that the electron density distribution of dusty plasma is parabolic model, variations of power reflection, transmission, and absorption coefficients with frequencies of the incident wave are calculated as the wave illuminates the nonuniform dusty plasma from different angles. The effects of incident angles, number density, and radius of the dust particles on propagation characteristics are discussed in detail. Numerical results show that the number density and radius of the dust particles have very little influences on reflection and transmission coefficients and have obvious effects on absorption coefficients. The terahertz wave has good penetrability in dusty plasma.
\end{abstract}

\section{Introduction}

Dusty plasma is a kind of ionized gas mixed with dust particles, and it is also called complex plasma. It exists in space environment widely, such as the interstellar cloud, planetary ring, and planetary magnetosphere, as well as in the fields of semiconductor science and materials science. Dust particles in dusty plasma affect the electromagnetic environments of near-earth's space and may cause important effects on space communications system. Dust particles in laboratory environments will produce pollution in the process of plasma etching of semiconductor chips. Due to the presence of dust particles, dusty plasma possesses many characteristics which are different from general plasma $[1,2]$. Since the beginning of 1980 s, the dusty plasma science has attracted the attention of a large number of scholars and has become an important branch of plasma physics.

At present, the interactions between high frequency electromagnetic wave and dusty plasma have become one of the research interests of many scholars. The dust particles in the plasma can change the physical properties of the general plasma through colliding with electrons, ions, charging, and electromagnetic force; for example, the dusty plasma system has unusual electromagnetic characteristics. Li et al. studied the absorbing effect of dusty particle on electromagnetic wave in plasma [3], as well as the scatter spectrum of radio wave by dusty plasma [4]. Guerra and Mendonça presented the Mie and Debye scattering characteristics in dusty plasma [5]. Havnes et al. provided important results on the relationship between charged dust particle and PMSE phenomenon [6,7]. Scales researched the electrodynamic structure of charged dust clouds in earth's middle atmosphere [8]. Shi et al. discussed the influence of charge and discharge process of dust particles on the dielectric constant and conductivity of dusty plasma [9] and gave the expression of the dielectric tensor of weakly ionized dusty plasma [10].

Many studies show that terahertz $(\mathrm{THz})$ wave has good penetrability in medium. The propagation characteristics of terahertz wave in dusty plasma will play an important role in the further analysis of dusty plasma effects on airground communication and may provide some theoretical basis for the dust particles pollution processing in the semiconductor laboratory. Li et al. [11] studied the propagation characteristics of THz wave propagation in plasma sheath and discussed the effects of incident angles and plasma collision frequencies on transmission parameters. In this paper, based 
on propagation matrix method, the propagation characteristics of oblique incident $\mathrm{THz}$ wave from nonuniform dusty plasma are studied. Numerical results of the power reflection, transmission, and absorption coefficients are given, and the effects of incident angles, density, and radius of dust particles are analyzed in detail.

\section{Basic Theory}

2.1. Complex Permittivity of Weakly Ionized Dusty Plasma. Dusty plasma is composed of dust particles, electrons, ions, and neutral molecules. The charging and discharging processes between electrons, ions, and dust particles will change the total currents of the dusty plasma system, thus affecting the conductivity and permittivity. Considering the effects of collision, charging, and discharging, [10] gave the relative complex permittivity of the dusty plasma as follows:

$$
\begin{aligned}
\varepsilon_{r}= & 1-\frac{\omega_{\mathrm{pe}}^{2}}{\omega^{2}+v_{\mathrm{eff}}^{2}}+\eta \frac{v_{\mathrm{ch}}+v_{\mathrm{eff}}}{\left(\omega^{2}+v_{\mathrm{ch}}^{2}\right)\left(\omega^{2}+v_{\mathrm{eff}}^{2}\right)} \\
& -j \frac{1}{\omega}\left[\frac{\omega_{\mathrm{pe}}^{2} v_{\mathrm{eff}}}{\left(\omega^{2}+v_{\mathrm{eff}}^{2}\right)}+\frac{\omega_{\mathrm{pe}}^{2} \omega_{d}\left(\omega^{2}-v_{\mathrm{ch}} v_{\mathrm{eff}}\right)}{\left(\omega^{2}+v_{\mathrm{ch}}^{2}\right)\left(\omega^{2}+v_{\mathrm{eff}}^{2}\right)}\right],
\end{aligned}
$$

where the notation $\omega_{d}$ denotes $\omega_{d}=4 \pi n_{d} r_{d}^{2} v_{\phi} ; n_{d}$ is the dust particles number density; $r_{d}$ is radius of a dust particle; $\omega_{\mathrm{pe}}=\left(4 \pi n_{e} e^{2} / m_{e}\right)^{1 / 2}$ is the electron plasma frequency; $\eta=e^{2} \pi r_{d}^{2} n_{e} n_{d} v_{\phi} / m_{e}\left(v_{\phi}=\omega / k\right)$ is the charging response factor; $n_{e}$ is the electron number density; $m_{e}$ is the electron mass; $\omega$ is the angular frequency; $k$ is the wavenumber of incident wave; and $v_{\text {eff }}$ is the effective collision frequency in dusty plasma.

The charging relaxation velocity [2] is

$$
\begin{aligned}
v_{\mathrm{ch}}= & -4 \pi r_{d} n_{e} e^{2}\left(\frac{K_{B} T}{2 \pi m_{e}}\right)^{1 / 2} \exp \left(-\frac{e^{2}}{K_{B} T r_{d}}\right) \\
& \cdot\left[\frac{1}{K_{B} T}+\frac{1}{K_{B} T+\left(e^{2} / r_{d}\right)}\right],
\end{aligned}
$$

where $K_{B}$ is the Boltzmann constant and $T$ is the electron temperature.

\subsection{Propagation Matrix Method of Oblique Incident $\mathrm{THz}$} Wave. Consider a plane terahertz wave incident on nonuniform plasma, as is shown in Figure 1. The time factor $\exp (i \omega t)$ is omitted. The incident electric field component is expressed as follows:

$$
\vec{E}^{i}=\widehat{e}_{y} E_{0} e^{-i \vec{k} \cdot \vec{r}}=\widehat{e}_{y} E_{0} e^{-i\left(k_{z} z+k_{x} x\right)},
$$

where $k_{z}=k \cos \theta_{i}, k_{x}=k \sin \theta_{i}$, and $\theta_{i}$ is the incident angle.

Nonuniform dusty plasma is divided into $n$ layers. There is the same electron density in each layer and the electromagnetic fields component in each layer can be expressed as the sum of incident and reflected fields. According to the boundary conditions of electromagnetic wave at different medium

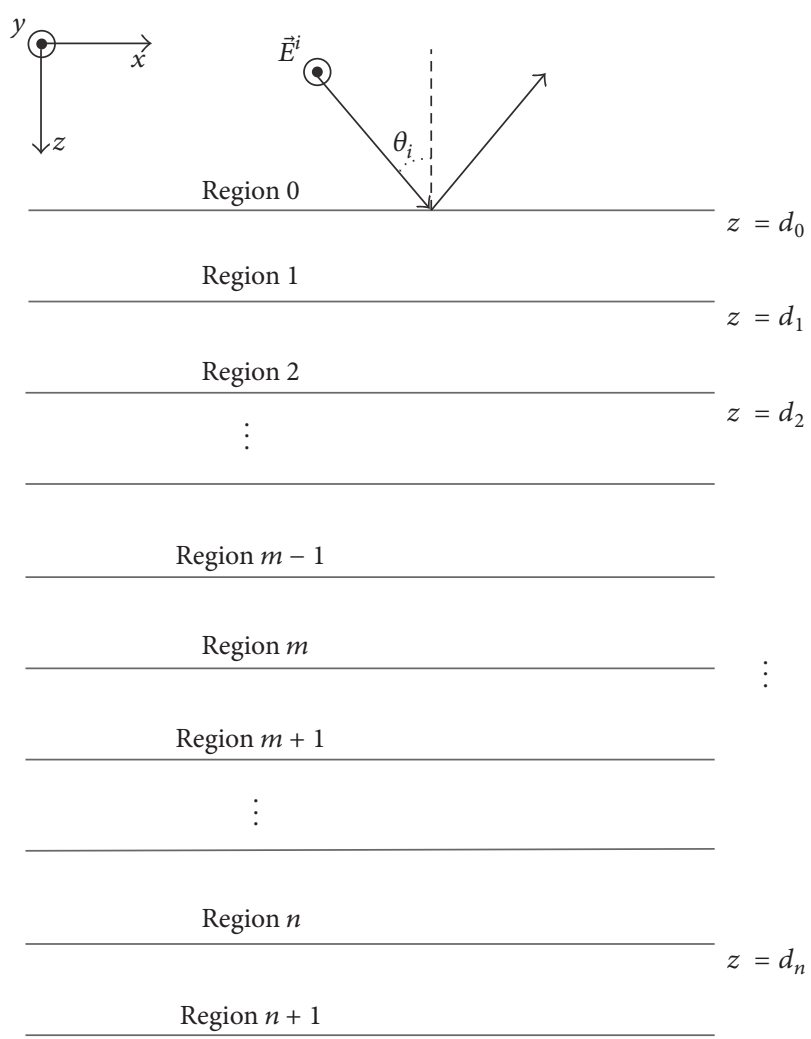

FIGURE 1: Schematic diagram of THz wave incident on nonuniform dusty plasma.

interfaces, the expression of total reflection coefficient in the 0 layer and the total transmission coefficients in the $n+1$ layer [12] can be obtained by propagation matrix method as follows:

$$
\left(\begin{array}{l}
A \\
D
\end{array}\right)=-\left(S_{g_{1}},-V_{n+1}\right)^{-1} \cdot S_{g_{2}},
$$

where $A$ and $D$ are the unknowns to be determined; $S_{g_{1}}$ and $S_{g_{2}}$ are the first and last column of $S_{g}$ matrix; and $S_{g}=$ $\left(\prod_{m=2}^{n} S_{m}\right) S_{1}$.

The matrix $S_{m}$ gives the incident and reflected field coefficients between the adjacent two layers as follows:

$$
\begin{aligned}
S_{m}= & \left(\begin{array}{cc}
e^{-i k_{z}^{(m)} d_{m}} & e^{i k_{z}^{(m)} d_{m}} \\
k_{z}^{(m)} e^{-i k_{z}^{(m)} d_{m}} & -k_{z}^{(m)} e^{i k_{z}^{(m)} d_{m}}
\end{array}\right)^{-1} \\
& \cdot\left(\begin{array}{cc}
e^{-i k_{z}^{(m-1)} d_{m}} & e^{i k_{z}^{(m-1) d_{m}}} \\
k_{z}^{(m-1)} e^{-i k_{z}^{(m-1)} d_{m}} & -k_{z}^{(m-1)} e^{i k_{z}^{(m-1)} d_{m}}
\end{array}\right) .
\end{aligned}
$$

$V_{n+1}$ is the matrix between incident and reflected field coefficients of the last interface.

$$
V_{n+1}=\frac{1}{2 k_{z}^{(n)}}\left(\begin{array}{c}
\left(k_{z}^{(n)}+k_{z}^{(n+1)}\right) e^{i\left(k_{z}^{(n)}-k_{z}^{(n+1)}\right) d_{n+1}} \\
\left(k_{z}^{(n)}-k_{z}^{(n+1)}\right) e^{-i\left(k_{z}^{(n)}+k_{z}^{(n+1)}\right) d_{n+1}}
\end{array}\right) .
$$

$k_{z}^{(j)}=k^{(j)} \cos \theta_{j}, j=2, \ldots, n$. 


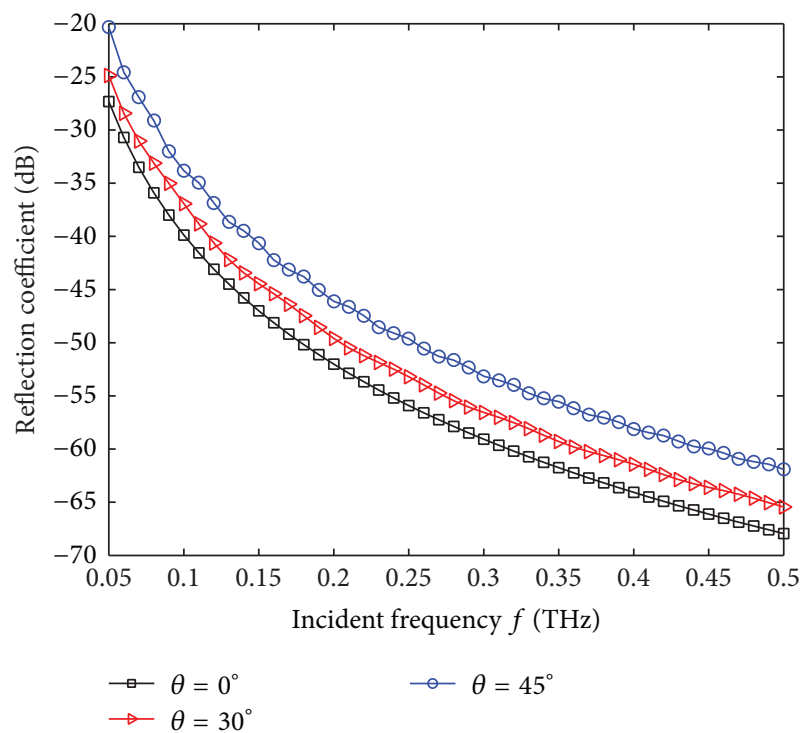

(a)

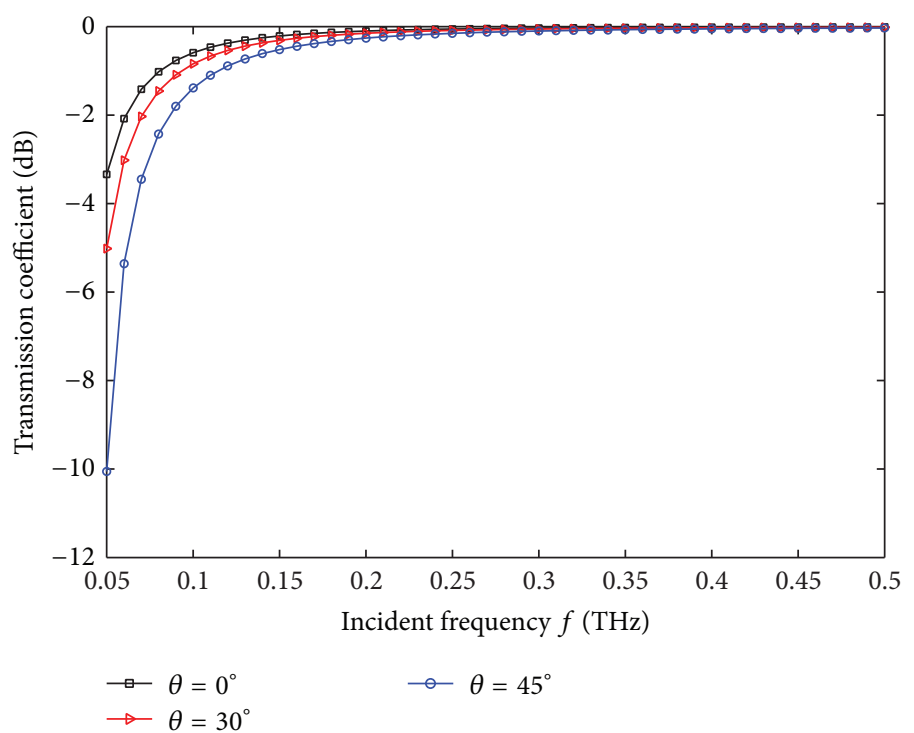

(b)

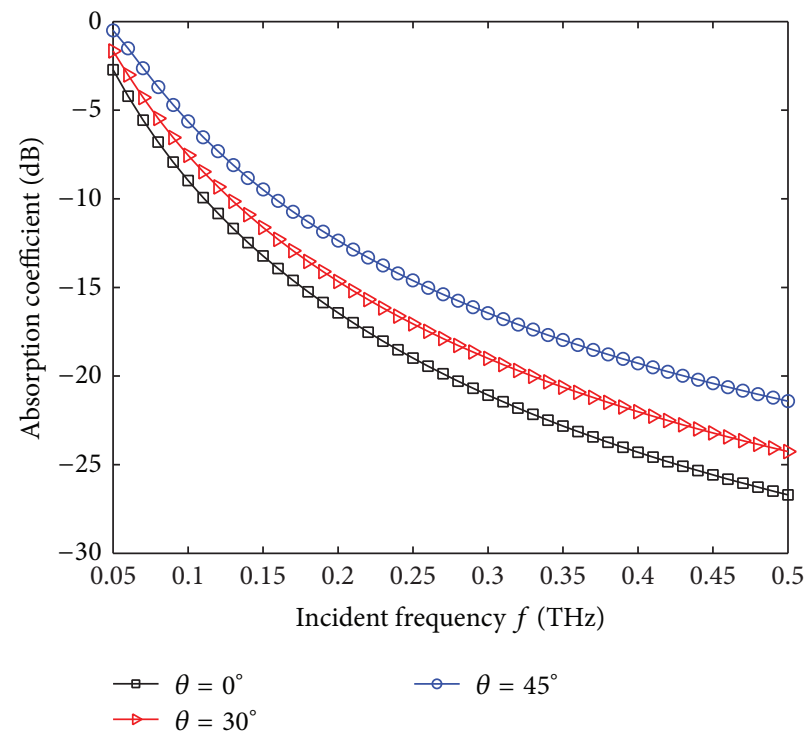

(c)

FIGURE 2: The effects of the incident angle on the reflection, transmission, and absorption coefficients when the wave is obliquely incident on the nonuniform dusty plasma. (a) Reflection coefficient; (b) transmission coefficient; (c) absorption coefficient.

\section{Numerical Results}

Based on the dielectric constant expression of weakly ionized dusty plasma and the propagation matrix method for oblique incidence, numerical results of the propagation characteristics of terahertz wave incident on nonuniform dusty plasma are given, and the effects of different parameters on the power reflection, transmission, and absorption coefficients are analyzed.

Figures 2(a)-2(c) show the variations of power reflection, transmission, and absorption coefficients with the frequency while $\mathrm{THz}$ wave illuminates dusty plasma at different angles. In the dusty plasma layer model, plasma parabolic model and certain dust particle number density are used. In Figure 2, the number density of dusty particles is $n_{d}=4 \times 10^{6} / \mathrm{cm}^{3}$, the radius of a dust particle is $r_{d}=5 \times 10^{-4} \mathrm{~cm}$, and electron temperature is $T=150 \mathrm{~K}$; the plasma is divided into 20 layers and parabolic model is used to describe the electron distribution as follows: $n_{e}=n_{0}\left(1-z^{2} / a^{2}\right), n_{0}=5 \times 10^{5} / \mathrm{cm}^{3}$; and the thickness of dusty plasma layer is $a=1.5 \mathrm{~cm}$. For the same incident frequency, reflection and absorption coefficients increase gradually with the increasing of incident angles; however, the transmission coefficients are just contrary. For the same incident angle, as the incident frequency increases, the reflection and absorption coefficients decrease gradually, while transmission coefficients increase gradually and are close to full transmission finally. The result shows that as the frequency of incident wave increases to $\mathrm{THz}$ wave band, 


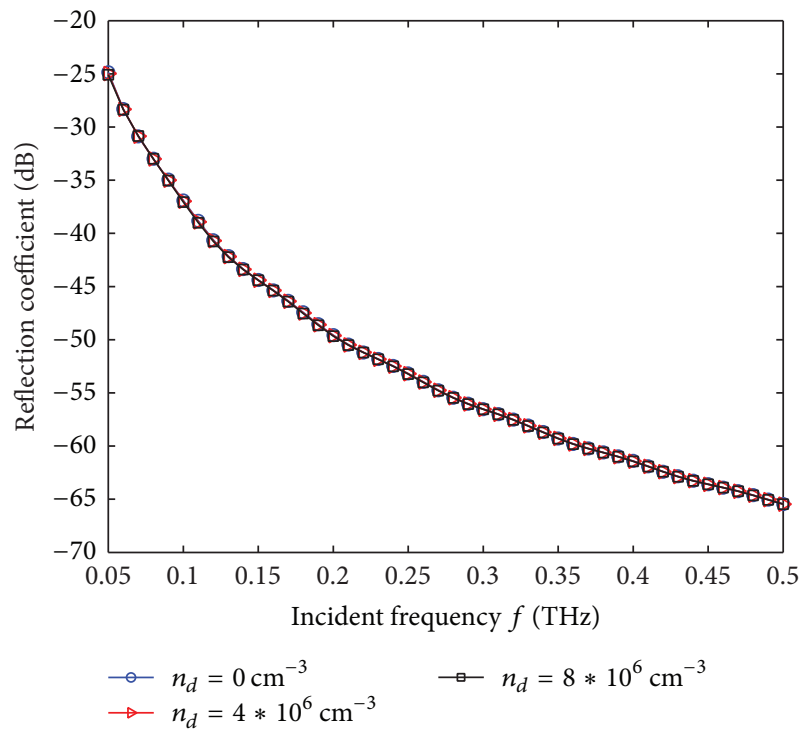

(a)

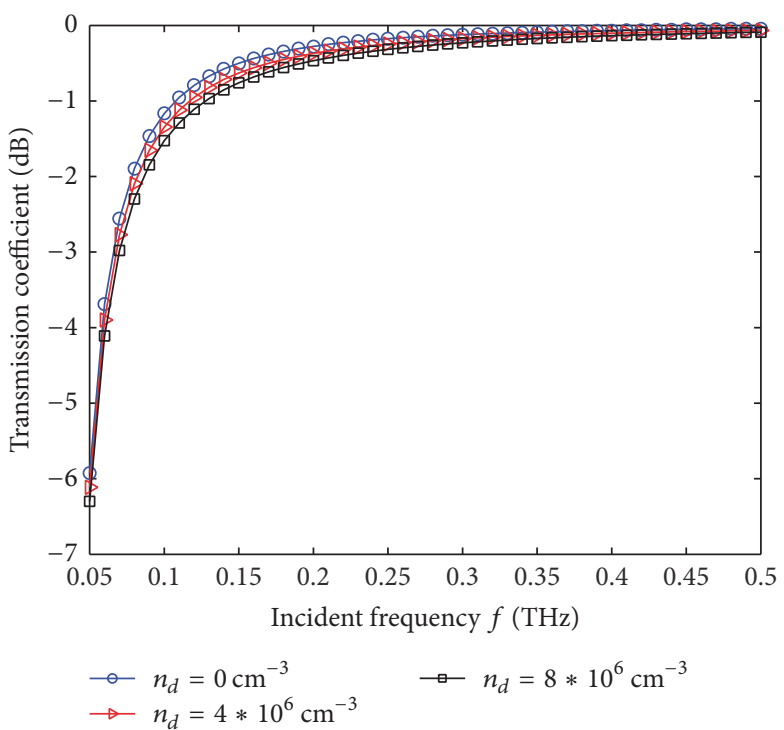

(b)

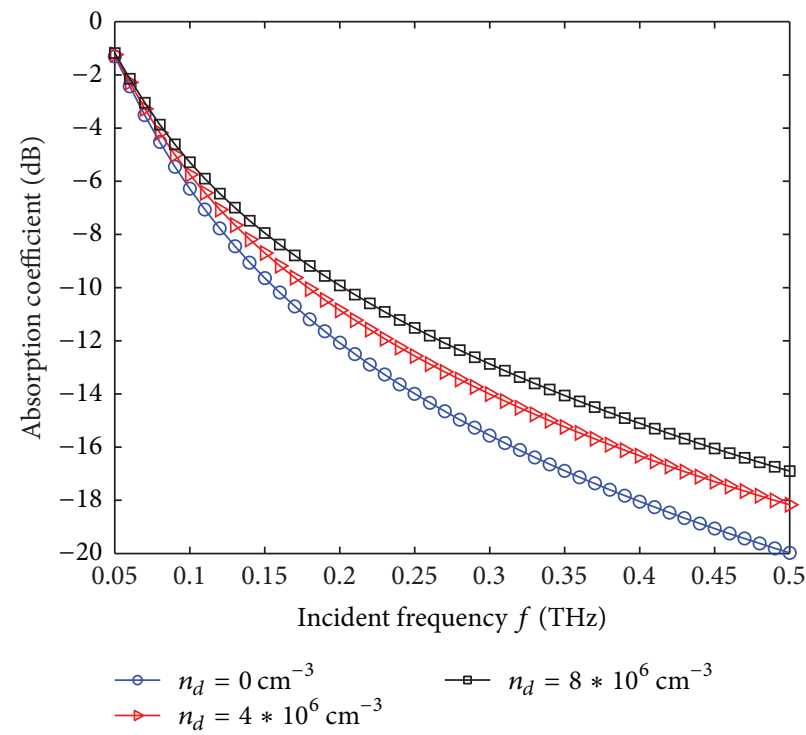

(c)

FIGURE 3: The effects of number density of dust particles on reflection, transmission, and absorption coefficients when THz wave is obliquely incident on nonuniform dusty plasma. (a) Reflection coefficients; (b) transmission coefficients; (c) absorption coefficients.

there is a good transmission performance of the nonuniform dusty plasma. This is in agreement with the notion that $\mathrm{THz}$ wave has a good penetrability.

Figures 3(a)-3(c) show the effects of dust particle number density on the reflection, transmission, and absorption coefficients. The calculation parameters are as follows: the radius of a dust particle is $r_{d}=5 \times 10^{-4} \mathrm{~cm}$; parabolic model is used to describe the electron distribution as follows: $n_{0}=$ $5 \times 10^{5} / \mathrm{cm}^{3}$; electron temperature is $T=150 \mathrm{~K}$; the thickness of dusty plasma layer is $1.5 \mathrm{~cm}$; and the incident angle is 30 degrees.

In Figure 3(a), the number density of dusty particles has smaller effects on the reflection coefficients; the results between cases showing that there is no dust particles and that the number density of dust particles is $n_{d}=8 \times 10^{6} / \mathrm{cm}^{3}$ are almost coincident. In Figure 3(b), as the number density of dust particles increases, within the $0.05-0.25 \mathrm{THz}$ bands, the transmission coefficients decrease gradually. However, as the frequency continues to increase, the transmission coefficients of three situations tend to $0 \mathrm{~dB}$ gradually, and the differences are not obvious. In Figure 3(c), for the same incident frequencies, absorption coefficients increase gradually with the increasing of number density of dust particles. Meanwhile, as the increasing of incident wave frequency, absorption coefficients increase quickly. For the same number density of dust particles, absorption coefficients decrease gradually with the increasing of incident wave frequencies.

Figures $4(\mathrm{a})-4(\mathrm{c})$ show the effects of radius of a dust particles on the reflection, transmission, and absorption coefficients. The calculation parameters in this figure are 


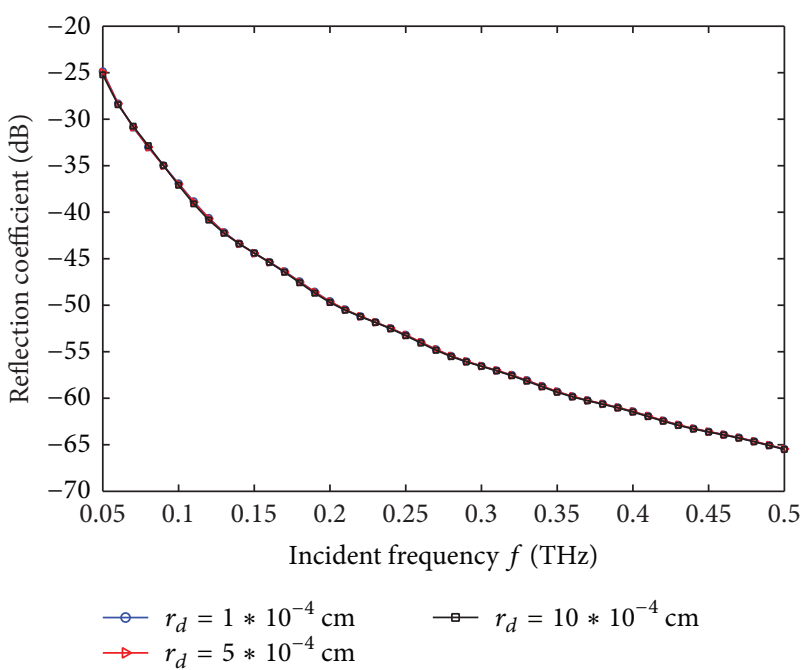

(a)

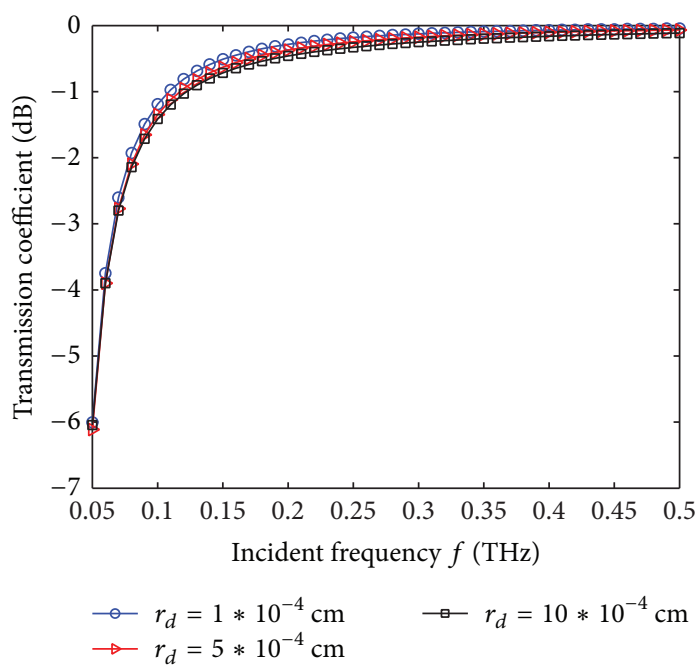

(b)

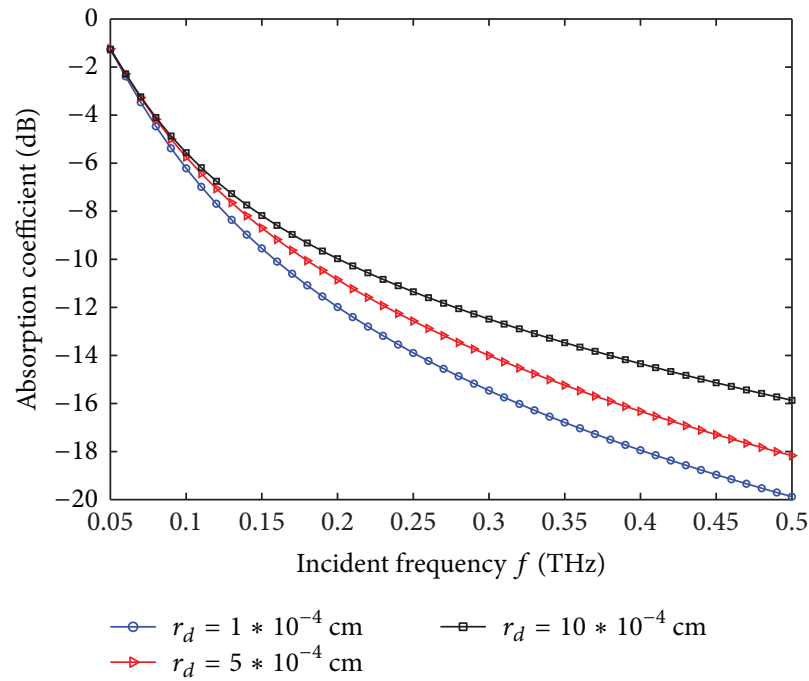

(c)

FIGURE 4: The effects of the radius of a dust particle on reflection, transmission, and absorption coefficients when THz wave is obliquely incident on nonuniform dusty plasma. (a) Reflection coefficients; (b) transmission coefficients; (c) absorption coefficients.

as follows: number density of dust particles is $n_{d}=4 \times$ $10^{-6} / \mathrm{cm}^{-3}$; parabolic model is used to describe the electron distribution as follows: $n_{0}=5 \times 10^{5} / \mathrm{cm}^{3}$; electron temperature is $T=150 \mathrm{~K}$; the thickness of dusty plasma layer is $1.5 \mathrm{~cm}$; and the incident angle is 30 degrees. For the dusty plasma that has the same number density of dust particles, as the radius of a dust particle increases, reflection coefficients change little; transmission coefficients change significantly in the range of 0.05-0.3 THz; and absorption coefficients decrease with the increasing of the dust particle radius.

\section{Conclusion}

The variations of power reflection, transmission, and absorption coefficients for case of $\mathrm{THz}$ wave obliquely incident on the nonuniform dusty plasma are analyzed by propagation matrix method. The effects of incident frequency, number density, and radius of dust particles on reflection, transmission, and absorption coefficients are presented numerically. The results show that the increasing of incident angles will increase the reflection and absorption coefficients and decrease the transmission coefficients; radius and number density of dust particles can affect absorption coefficients obviously, influence the transmission coefficients in a certain frequency band range, and have little effects on reflection coefficients. Results in this paper may provide a certain theoretical basis for further analysis and study of propagation characteristics of $\mathrm{THz}$ wave in the middle atmosphere in the future air-ground communication.

\section{Competing Interests}

The authors declare that they have no competing interests. 


\section{Acknowledgments}

This work was supported by National Natural Science Foundation of China [Grants nos. 61475123 and 61571355] and The Project Supported by Natural Science Basic Research Plan in Shaanxi Province of China [Grant no. 2016JQ4015].

\section{References}

[1] J. X. Ma, “Dusty plasmas," Physics, vol. 3, pp. 244-250, 2006.

[2] P. K. Shukla and A. A. Mamun, Introduction to Dusty Plasma Physics, IOP Publishing, London, UK, 2002.

[3] F. Li, L. L. Li, and Q. Sui, "Absorbing effect of dusty particle on Electromagnetic wave in plasma," Science in China E, vol. 7, pp. 832-840, 2004.

[4] F. Li and O. Havnes, "Scatter spectrum of Radio wave by a Dusty plasma," Journal of Electronics, vol. 5, pp. 679-685, 1999.

[5] R. Guerra and J. T. Mendonça, "Mie and Debye scattering in dusty plasmas," Physical Review E-Statistical Physics, Plasmas, Fluids, and Related Interdisciplinary Topics, vol. 62, no. 1, pp. 1190-1201, 2000.

[6] O. Havnes, F. Melandsø, C. L. Hoz, T. K. Aslaksen, and T. Hartquist, "Charged dust in the Earth's mesopause: effects on radar backscatter," Physica Scripta, vol. 45, no. 5, pp. 535-544, 1992.

[7] O. Havnes, T. Aslaksen, and A. Brattli, "Charged dust in the earth's Middle Atmosphere," Physica Scripta T, vol. 89, pp. 133137, 2001.

[8] W. A. Scales and G. Ganguli, "Electrodynamic structure of charged dust clouds in the earth's middle atmosphere," New Journal of Physics, vol. 1, pp. 1-15, 2004.

[9] Y.-X. Shi, D.-B. Ge, and J. Wu, "Influence of charge and discharge processes of dust particles on the dust plasma conductivity," Acta Physica Sinica, vol. 55, no. 10, pp. 5318-5324, 2006.

[10] Y.-X. Shi, J. Wu, and D.-B. Ge, "The research on the dielectric tensor of weakly ionized dust plasma," Acta Physica Sinica, vol. 58, no. 8, pp. 5507-5512, 2009.

[11] S. T. Li, J. Li, and Z. B. Zhu, "Studies on terahertz wave propagation in plasma sheath," Journal of Terahertz Science and Electronic Information Technology, vol. 2, pp. 203-214, 2015.

[12] B. J. Hu, G. Wei, and L. Sheng, "SMM analysis of reflection, absorption, and transmission from nonuniform magnetized plasma slab," IEEE Transactions on Plasma Science, vol. 27, no. 4, pp. 1131-1136, 1999. 


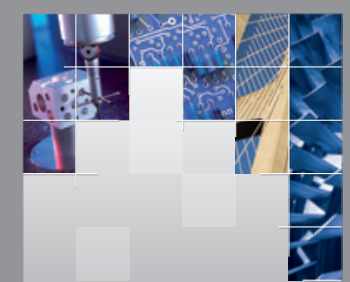

\section{Enfincering}
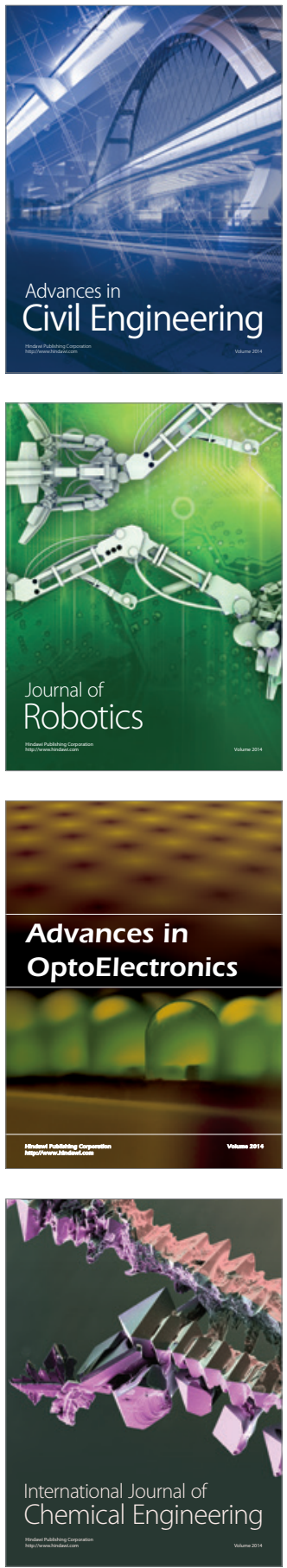

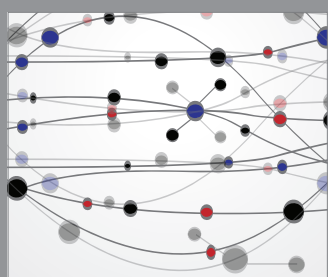

The Scientific World Journal

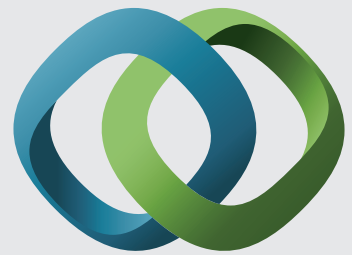

\section{Hindawi}

Submit your manuscripts at

http://www.hindawi.com
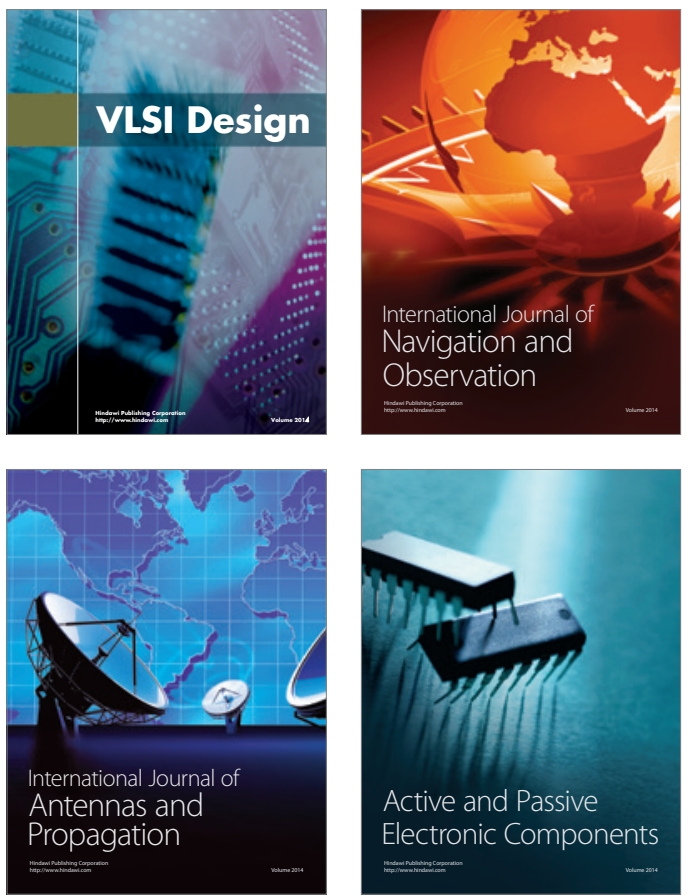
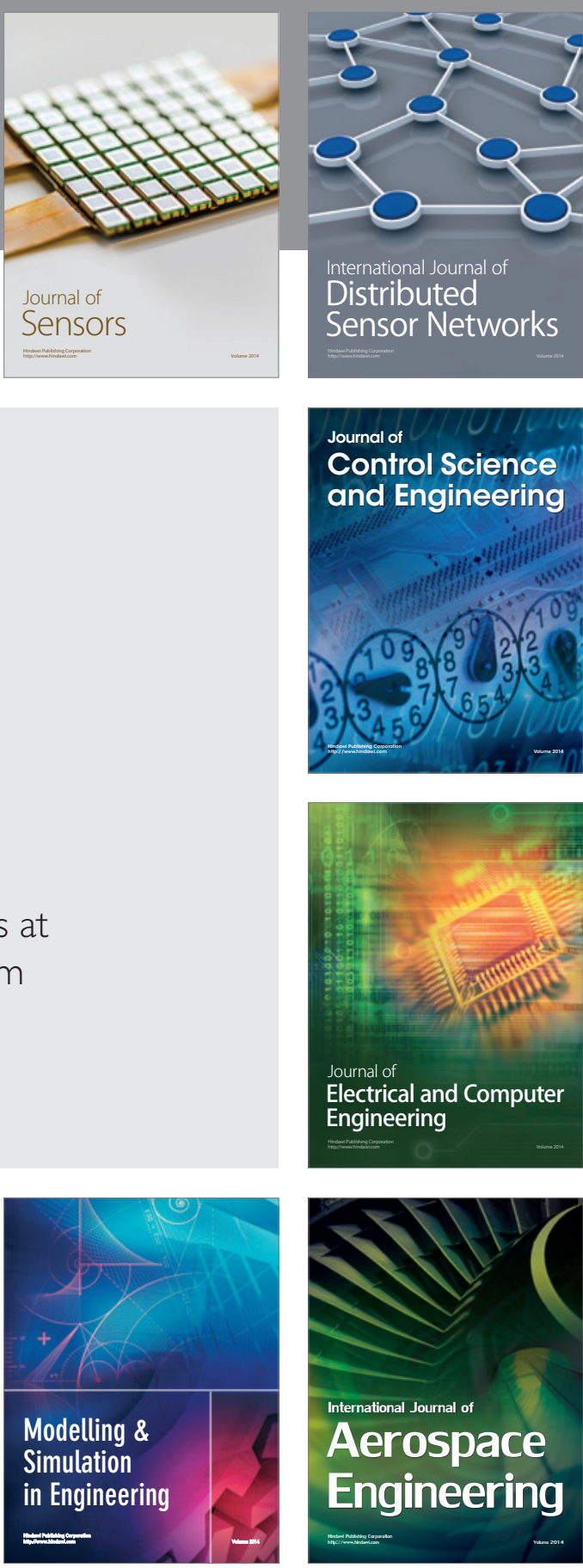

International Journal of

Distributed

Sensor Networks

Journal of

Control Science

and Engineering
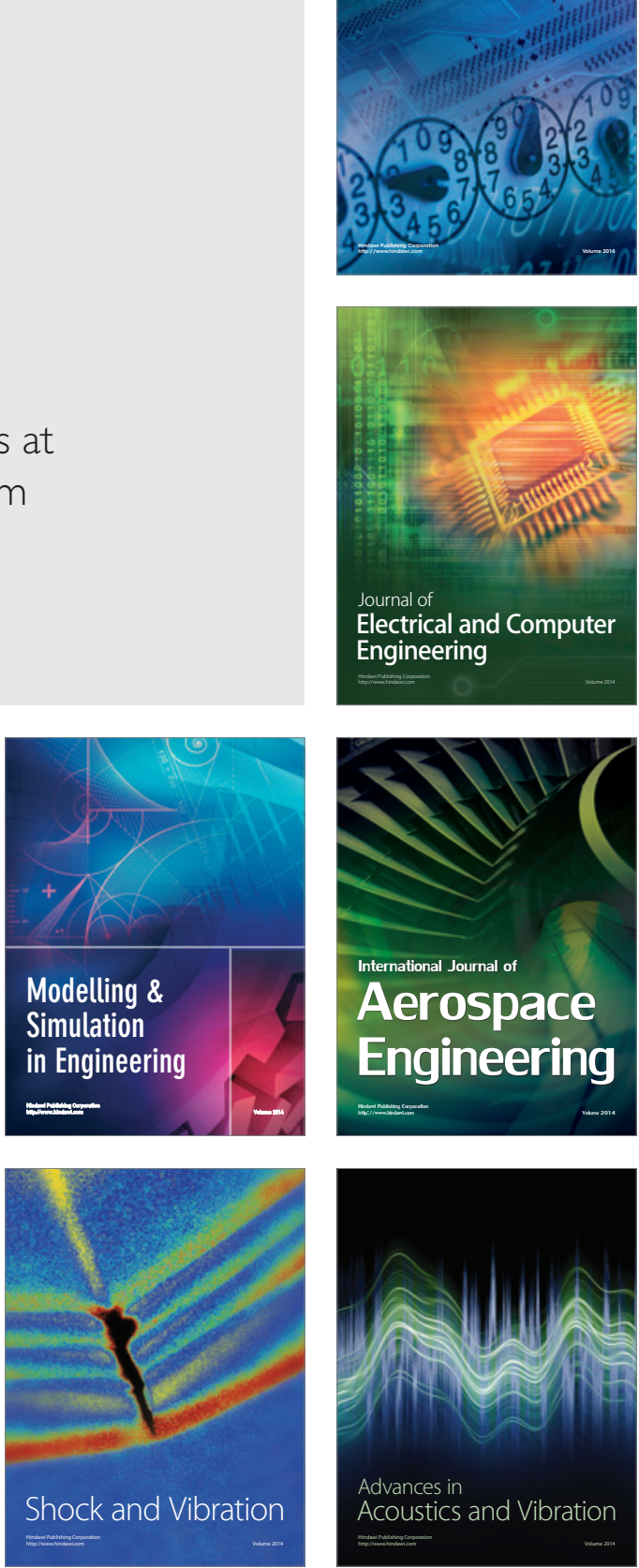